\title{
Charge-Carrier Transport in Thin Film Solar Cells: New Formulation
}

\author{
Jesús E. Velazquez-Perez ${ }^{1}$ and Yuri G. Gurevich ${ }^{2}$ \\ ${ }^{1}$ Departamento de Física Aplicada, Universidad de Salamanca, 37008 Salamanca, Spain \\ ${ }^{2}$ Departamento de Física, CINVESTAV del I.P.N., Apartado Postal 14-740, 07000 Mexico, DF, Mexico
}

Correspondence should be addressed to Jesús E. Velazquez-Perez, js@usal.es

Received 4 July 2011; Accepted 28 August 2011

Academic Editor: Leonardo Palmisano

Copyright (C) 2011 J. E. Velazquez-Perez and Y. G. Gurevich. This is an open access article distributed under the Creative Commons Attribution License, which permits unrestricted use, distribution, and reproduction in any medium, provided the original work is properly cited.

\begin{abstract}
Solar cells rely on photogeneration of charge carriers in $\mathrm{p}-\mathrm{n}$ junctions and their transport and subsequent recombination in the quasineutral regions. A number of basic issues concerning the physics of the operation of solar cells still remain obscure. This paper discusses some of those unsolved basic problems. In conventional solar cells, recombination of photogenerated charge carriers plays a major limiting role in the cell efficiency. High quality thin-film solar cells may overcome this limit if the minority diffusion lengths become large as compared to the cell dimensions, but, strikingly, the conventional model fails to describe the cell electric behavior under these conditions. A new formulation of the basic equations describing charge carrier transport in the cell along with a set of boundary conditions is presented. An analytical closed-form solution is obtained under a linear approximation. In the new framework given, the calculation of the open-circuit voltage of the solar cell diode does not lead to unphysical results.
\end{abstract}

\section{Introduction}

Charge carrier transport underlies the electrical behavior of any semiconductor device and, in particular, of solar cells. Despite efforts made over the years to correctly model charge-carrier transport in semiconductors, some important questions still remain unanswered. These questions need to be addressed in order to correctly model present and future devices.

One of these open questions is how to model carrier recombination. The mathematical expression routinely used to model the recombination rate [1] is basically incorrect. It has recently been demonstrated that it contradicts Maxwell's equations, and hence a new corrected model must be developed $[2,3]$. This problem was partly addressed in previous works (see, e.g., [4]). Recombination is a key feature when describing carrier transport in solar cells because it strongly affects the electrical response of the semiconductor at all levels of external excitation. A remark that must be made about the need for a correct modeling of recombination is that in devices operating under a strong excitation regime (the operation of solar cells lies in this regime), the importance of a correct formulation of the recombination terms is even more important.

Since in general the set of Poisson and transport equations cannot be solved analytically, some simplifications must be introduced to obtain a closed-form solution. One approximation commonly used to solve this system of equations is the assumption of quasineutrality (QN) [5]. The use of the QN approximation is acceptable if the sample's and the diffusion lengths are both larger than the Debye length. Although QN has been routinely used in semiconductor device modeling for many years, the role of space charge in the formation of the current-voltage $(I-V)$ characteristic in a semiconductor is still controversial.

A very important question is the choice of the boundary conditions used when solving the carrier-transport equations. It should be noted that the expressions commonly used are valid only for semiconductor devices operating in open-circuit conditions (see, e.g., [6]). Since in normal operation a current flows at the terminals, the widespread use of boundary conditions for open-circuit conditions is 
incorrect. For closed-circuit conditions, a different set of boundary conditions needs to be derived. This problem has only been addressed in the last few years [7-9].

All the abovementioned issues need to be addressed when modeling any semiconductor devices. Thin-film solar cells are one of the strongest technologies in the steadily growing photovoltaic market [10]. In this paper we will address a very basic problem encountered when modeling cells with large values of the minority diffusion lengths.

\section{Problem Statement}

The commonly accepted electrical model for the current density-voltage characteristic $(J-V)$ of an ideal p-n junction is [11]

$$
J=J_{0}\left(e^{V / V_{T}}-1\right)
$$

where $J$ is the diode current density, $V$ is the bias voltage, $V_{T}$ is the thermal voltage, and $J_{0}$ the reverse-saturation current density that for a long abrupt diode is

$$
J_{0}=V_{T}\left(\frac{\sigma_{\mathrm{p}}^{\mathrm{n}}}{L_{D}^{\mathrm{n}}}+\frac{\sigma_{\mathrm{n}}^{\mathrm{p}}}{L_{D}^{\mathrm{p}}}\right),
$$

$\sigma_{\mathrm{n}}^{\mathrm{p}}\left(\sigma_{\mathrm{p}}^{\mathrm{n}}\right)$ is the electron (hole) conductivity of minority carriers in the p-side (n-side) of a junction diode; $L_{D}^{\mathrm{n}}$ and $L_{D}^{\mathrm{p}}$ are, respectively, the hole and electron minority carrier diffusion lengths of minority carriers in $\mathrm{n}$ - and $\mathrm{p}$ - sides defined as

$$
L_{D}^{\mathrm{n}}=\sqrt{D_{\mathrm{p}} \tau_{\mathrm{p}}}, \quad L_{D}^{\mathrm{p}}=\sqrt{D_{\mathrm{n}} \tau_{\mathrm{n}}}
$$

$D_{\mathrm{n}}\left(D_{\mathrm{p}}\right)$ is the electron (hole) diffusivity and $\tau_{\mathrm{n}}\left(\tau_{\mathrm{p}}\right)$ is the electron (hole) minority carrier lifetime.

Equation (1) may be rewritten for a solar cell under illumination as [12]

$$
-J=J_{0}\left(e^{V / V_{T}}-1\right)+J_{I}
$$

where $J_{I}$ is the photocurrent and $J$ is additionally related to the generated voltage $V$ through the external resistive load $(R)$ in the circuit and the solar cell area $(S)$ as shown in Figure 1:

$$
J=\frac{V}{R S}
$$

For the sake of simplicity, let us examine the photocurrent generated in a slab of semiconductor of thickness $L$ with incident light at $x=0$. $J_{I}$ may be obtained as the integral of the light intensity across the device $I(x)$ as

$$
\left|J_{I}\right|=q \alpha \beta \int_{0}^{L} I(x) d x,
$$

where $q$ is the absolute value of the electron charge, $\alpha$ is the absorption coefficient of light, and $\beta$ is the quantum efficiency,

$$
\beta=\frac{\alpha-\alpha_{0}}{\alpha}
$$

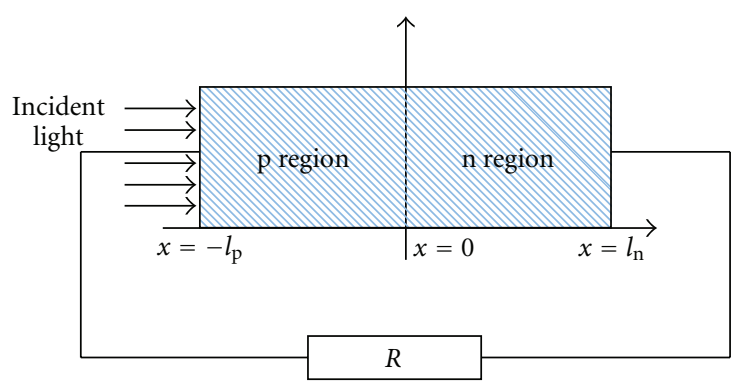

Figure 1: Solar cell diode with an external resistor load.

The light intensity inside the semiconductor slab will decrease following the law:

$$
I=I_{0} e^{-\alpha x}
$$

where $I_{0}$ is the light intensity (number of incident by photons per unit time and unit area) at the plane of the incident light $(x=0)$ and the carrier generation rate due to photonabsorption can be written as

$$
g=\left(\alpha-\alpha_{0}\right) I_{0} e^{-\alpha x}
$$

The set of (4)-(9) may be extended or augmented with other equations for a finer modeling of a real technology [13], but this set constitutes the fundamental framework to describe the electrical behaviour of any diode-based solar cell. Let us assume a one-dimensional problem for a singlediode solar cell such as the one given in Figure 1. According to the values of diffusion lengths in $\mathrm{Si}$, in a c-Si thin-film solar cell, or in a-Si ones of nanometer dimensions [14], the following inequality will hold:

$$
l_{\mathrm{n}, \mathrm{p}} \ll L_{D}^{\mathrm{n}, \mathrm{p}} .
$$

From (10) it follows that $L_{D}^{\mathrm{n}, \mathrm{p}} \rightarrow \infty$ and $\tau_{\mathrm{n}, \mathrm{p}} \rightarrow \infty$; therefore, recombination is negligible and according to (2) $J_{0} \rightarrow$ 0 and, moreover, from (4), the basic model leads to the unphysical result (see Figure $2(\mathrm{~b})$ ): $J \approx-J_{I}$, i.e., the $\mathrm{p}-\mathrm{n}$ junction behaves as a current-source that translates photoexcitation into current at any value of the diode-voltage independently of the $\mathrm{p}-\mathrm{n}$ junction properties.

From the above it follows that the framework of basic equations conventionally used to model solar cells fails in high-quality thin-film solar cells [15]. Regardless of its apparent simplicity, since this framework constitutes the foundations of any electrical modeling of a solar cell diode, the discussed problem will persist in any other model that one can build; therefore, it is crucial to identify the origin of the problem and modify the basic model to build a new framework free from unphysical errors. In this paper we will present a new model and we will analytically show in a simple case that this new framework does not fail to correctly describe the limiting case of a thin-film solar cell.

\section{New Model for Thin-Film Solar Cells}

Let us assume once again that inequality of (10) holds and, accordingly, the solar cell recombination is negligible. The 


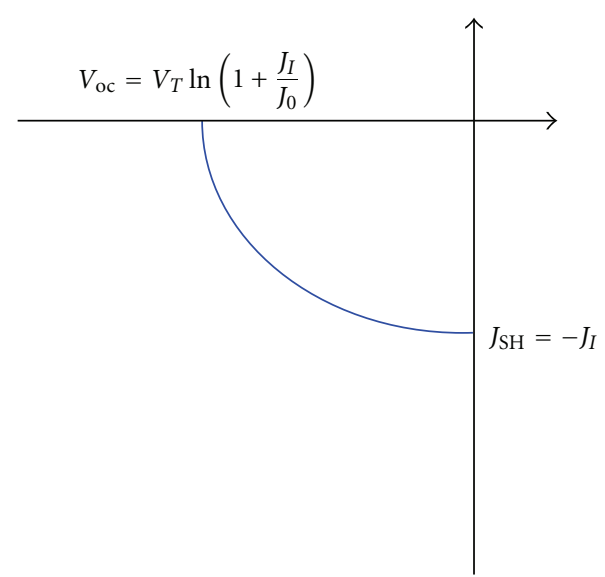

(a)

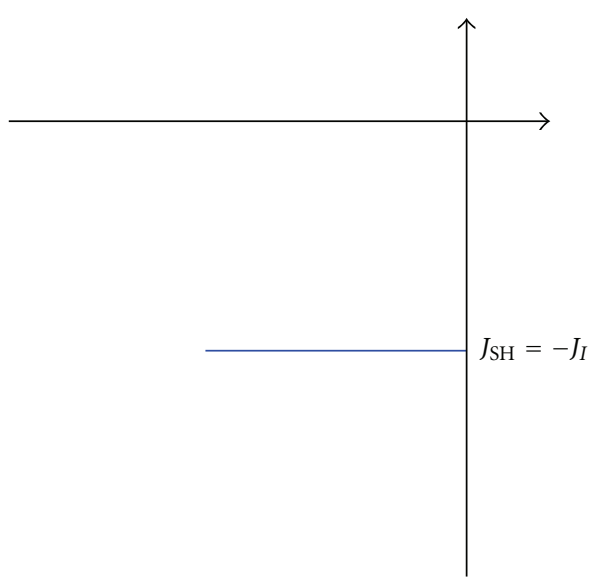

(b)

FIGURE 2: $J-V$ characteristic of an ideal solar cell with the short- and open-circuit expressions for the axis-intercept points $V_{\mathrm{oc}}$ and $J_{\mathrm{SH}}$, respectively, (a). $J-V$ characteristic in the limiting case of a thin-film cell $l_{\mathrm{n}, \mathrm{p}} \ll L_{D}^{\mathrm{n}, \mathrm{p}}$ (b).

macroscopic description of the transport of nonequilibrium charge is done with the continuity equations for the electron $\left(j_{\mathrm{n}}\right)$ and hole $\left(j_{\mathrm{p}}\right)$ current densities [3]:

$$
\begin{aligned}
& \frac{\partial \mathrm{n}}{\partial t}=g_{\mathrm{n}}+\frac{1}{e} \operatorname{div} j_{\mathrm{n}}-R_{\mathrm{n}}, \\
& \frac{\partial \mathrm{p}}{\partial t}=g_{\mathrm{p}}-\frac{1}{e} \operatorname{div} j_{\mathrm{p}}-R_{\mathrm{p}} .
\end{aligned}
$$

In a stationary case and one-dimensional problem along the $x$-direction, normal to the $\mathrm{p}$-n junction plane as in Figure 1, (11) becomes

$$
\begin{gathered}
\frac{d j_{\mathrm{n}}}{d x}=q\left(R_{\mathrm{n}}-g_{\mathrm{n}}\right), \\
\frac{d j_{\mathrm{p}}}{d x}=-q\left(R_{\mathrm{p}}-g_{\mathrm{p}}\right) .
\end{gathered}
$$

Since volume recombination is negligible $R_{\mathrm{n}}=R_{\mathrm{p}}=0$ and, accordingly, $g_{\mathrm{n}}=g_{\mathrm{p}}=g(x)$, that is, the recombination is band-to-band and the continuity equations reduce to

$$
\begin{gathered}
\frac{d j_{\mathrm{n}}}{d x}=-q g(x), \\
\frac{d j_{\mathrm{p}}}{d x}=q g(x) .
\end{gathered}
$$

Additionally, it can be shown that in absence of recombination and under band-to-band generation, the density of charge in any impurity level remains constant [15], under all the above conditions the QN approximation reduces to $\delta \mathrm{n}=$ $\delta \mathrm{p}$. In a general case, for a nondegenerate semiconductor, in a linear approximation $\delta \mathrm{n}$ and $\delta \mathrm{p}$ can be written in terms of the variations in their respective chemical potentials $\left(\mu_{\mathrm{n}}\right.$ and $\left.\mu_{\mathrm{p}}\right)$ as [16]

$$
\begin{aligned}
& \delta \mathrm{n}=\frac{\mathrm{n}_{0}}{T_{0}} \delta \mu_{\mathrm{n}}, \\
& \delta \mathrm{p}=\frac{\mathrm{p}_{0}}{T_{0}} \delta \mu_{\mathrm{p}},
\end{aligned}
$$

where the subscript 0 stand for equilibrium value of the magnitude, and using the QN approximation the following relationship between the deviations from equilibrium of the chemical potentials holds

$$
\delta \mu_{\mathrm{p}}=\frac{\mathrm{n}_{0}}{\mathrm{p}_{0}} \delta \mu_{\mathrm{n}}
$$

In order, to further simplify the mathematical problem, while ensuring no loss of generality of the physical one, we will assume a step-like profile for the light absorption instead of the model in (8):

$$
I= \begin{cases}I_{0} & -l_{\mathrm{p}}<x<0, \\ 0 & 0<x<l_{\mathrm{n}} .\end{cases}
$$

Using (9) and (16), we may write the continuity equations, (13), as

$$
\begin{array}{cc}
\frac{d j_{\mathrm{n}}^{\mathrm{p}}}{d x}=-q g_{0}, & \frac{d j_{\mathrm{p}}^{\mathrm{p}}}{d x}=q g_{0}, \\
\frac{d j_{\mathrm{n}}^{\mathrm{n}}}{d x}=0, & \frac{d j_{\mathrm{p}}^{\mathrm{n}}}{d x}=0 .
\end{array}
$$

Superscripts in current densities in (17) indicate the doping type of the region. The current densities may be written as [3]

$$
\begin{aligned}
& j_{\mathrm{n}}^{\mathrm{n}}=-\sigma_{\mathrm{n}}^{\mathrm{n}}\left(\frac{d \delta \varphi^{\mathrm{n}}}{d x}-\frac{1}{q} \frac{d \delta \mu_{\mathrm{n}}^{\mathrm{n}}}{d x}\right), \\
& j_{\mathrm{p}}^{\mathrm{n}}=-\sigma_{\mathrm{p}}^{\mathrm{n}}\left(\frac{d \delta \varphi^{\mathrm{n}}}{d x}+\frac{1}{q} \frac{d \delta \mu_{\mathrm{p}}^{\mathrm{n}}}{d x}\right), \\
& j_{\mathrm{n}}^{\mathrm{p}}=-\sigma_{\mathrm{n}}^{\mathrm{p}}\left(\frac{d \delta \varphi^{\mathrm{p}}}{d x}-\frac{1}{q} \frac{d \delta \mu_{\mathrm{n}}^{\mathrm{p}}}{d x}\right), \\
& j_{\mathrm{p}}^{\mathrm{p}}=-\sigma_{\mathrm{p}}^{\mathrm{p}}\left(\frac{d \delta \varphi^{\mathrm{p}}}{d x}+\frac{1}{q} \frac{d \delta \mu_{\mathrm{p}}^{\mathrm{p}}}{d x}\right),
\end{aligned}
$$


where we introduced the electric potential $(\varphi)$. To solve (17) with (14), (15), and (18), we need to determine and impose enough boundary conditions at the semiconductor interfaces [8]. We will assume ideal metallic (ohmic) contacts placed at $x=-l_{\mathrm{p}}$ and at $x=l_{\mathrm{n}}$; therefore, the excess of carries are null:

$$
\delta n^{\mathrm{p}}\left(-l_{\mathrm{p}}\right)=\delta p^{\mathrm{p}}\left(-l_{\mathrm{p}}\right)=0, \quad \delta n^{\mathrm{n}}\left(l_{\mathrm{n}}\right)=\delta p^{\mathrm{n}}\left(l_{\mathrm{n}}\right)=0 .
$$

And, according to (19), we obtain the following four boundary conditions for the chemical potentials at the metal-semiconductor contacts:

$$
\delta \mu_{\mathrm{n}}^{\mathrm{p}}\left(-l_{\mathrm{p}}\right)=\delta \mu_{\mathrm{p}}^{\mathrm{p}}\left(-l_{\mathrm{p}}\right)=0, \quad \delta \mu_{\mathrm{n}}^{\mathrm{n}}\left(l_{\mathrm{n}}\right)=\delta \mu_{\mathrm{p}}^{\mathrm{n}}\left(l_{\mathrm{n}}\right)=0 .
$$

Two additional boundary conditions may be imposed on the electric potential at the same metal-semiconductor contacts:

$$
\delta \varphi^{\mathrm{p}}\left(-l_{\mathrm{p}}\right)=0, \quad \delta \varphi^{\mathrm{n}}\left(l_{\mathrm{n}}\right)=V .
$$

At the interface of the two semiconductor regions, since there is no recombination, we may write two additional boundary conditions at $x=0[8]$ :

$$
j_{\mathrm{n}}^{\mathrm{n}}(0)=j_{\mathrm{n}}^{\mathrm{p}}(0), \quad j_{\mathrm{p}}^{\mathrm{n}}(0)=j_{\mathrm{p}}^{\mathrm{p}}(0) .
$$

Two more boundary conditions are

$$
\begin{aligned}
& \delta \varphi^{\mathrm{p}}(0)-\frac{\delta \mu_{\mathrm{n}}^{\mathrm{p}}(0)}{q}=\delta \varphi^{\mathrm{n}}(0)-\frac{\delta \mu_{\mathrm{n}}^{\mathrm{n}}(0)}{q}, \\
& \delta \varphi^{\mathrm{p}}(0)+\frac{\delta \mu_{\mathrm{p}}^{\mathrm{p}}(0)}{q}=\delta \varphi^{\mathrm{n}}(0)+\frac{\delta \mu_{\mathrm{p}}^{\mathrm{n}}(0)}{q} .
\end{aligned}
$$

Using the obtained boundary conditions from the continuity of the Fermi quasilevels it is straightforward to solve the linear system of equations for the current densities (18) and obtain the solution

$$
J\left(\frac{l_{\mathrm{n}}}{\sigma_{\mathrm{p}}^{\mathrm{n}}} \frac{l_{\mathrm{p}}^{\mathrm{p}}}{\sigma_{i}^{\mathrm{p}}}\right)+V\left(\frac{l_{\mathrm{p}}}{\sigma_{\mathrm{n}}^{\mathrm{p}}}+\frac{l_{\mathrm{n}}}{\sigma_{\mathrm{p}}^{\mathrm{n}}}\right)+\frac{q g_{0}}{2}\left(\frac{l_{\mathrm{p}}^{2} l_{\mathrm{n}}}{\sigma_{\mathrm{n}}^{\mathrm{p}} \sigma_{\mathrm{p}}^{\mathrm{n}}}\right)=0 .
$$

In the third term of (24), we may identify the photocurrent density created under illumination

$$
J_{I}=\frac{q g_{0}}{2} l_{\mathrm{p}} .
$$

Since we are under a small voltage-/current-linear approximation with the aim of obtaining an analytical closed form for the $J-V(24)$, we must compare the obtained equation with a linearization of (4):

$$
J_{I}+V\left(\frac{J_{0}}{V_{T}}\right)=-J
$$

By rewriting (24) in a similar form we obtain

$$
J_{I}+V\left(\frac{l_{\mathrm{p}}}{\sigma_{\mathrm{n}}^{\mathrm{p}}}+\frac{l_{\mathrm{n}}}{\sigma_{\mathrm{p}}^{\mathrm{n}}}\right)\left(\frac{\sigma_{\mathrm{n}}^{\mathrm{p}} \sigma_{\mathrm{p}}^{\mathrm{n}}}{l_{\mathrm{p}} l_{\mathrm{n}}}\right)=-J .
$$

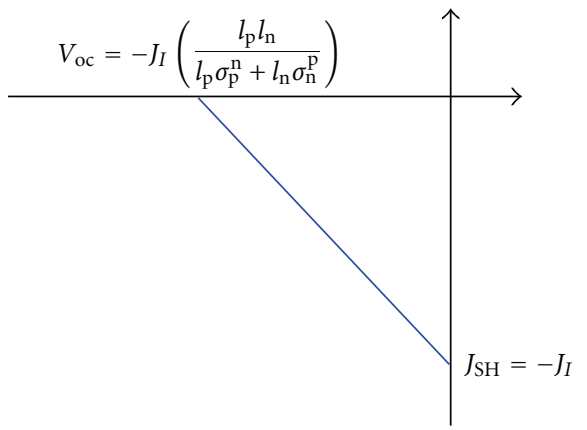

FIgURE 3: $J-V$ characteristic of an ideal thin-film solar cell with the new expression for the $x$ axis-intercept point $V_{\text {oc }}$.

This is a main result of this work, identifying the second terms in the left hand side of (26) and (27), we obtain a new expression for the current density $J_{0}$ :

$$
J_{0}=\left(\frac{l_{\mathrm{p}}}{\sigma_{\mathrm{n}}^{\mathrm{p}}}+\frac{l_{\mathrm{n}}}{\sigma_{\mathrm{p}}^{\mathrm{n}}}\right)\left(\frac{\sigma_{\mathrm{n}}^{\mathrm{p}} \sigma_{\mathrm{p}}^{\mathrm{n}}}{l_{\mathrm{p}} l_{\mathrm{n}}}\right) V_{T} .
$$

This new expression does not exhibit an unphysical behavior $\left(J_{0} \rightarrow \infty\right)$ in thin-film solar cells (Figure 2(b)). Figure 3 gives the two axis-intercept points $\left(V_{\mathrm{Oc}}\right.$ and $\left.J_{\mathrm{SH}}\right)$ of the $J-V$ characteristic, it can be trivially verified that the $x$-intercept point $\left(V_{\text {oc }}\right)$ will remain at a finite value independently of the minority carriers diffusion lengths at each side of the junction and the diode dimensions. Since the analytical model presented in this paper was obtained by a linearization of the full model and some other approximations have been used to simplify the mathematical problem, the large-signal $J-V$ is not properly obtained (Figure 3 ), but the framework of transport equations and boundary conditions can be solved numerically to study any real solar cell diode.

\section{Conclusions}

Solar cells rely on photogeneration of charge carriers in $p-n$ junctions and their subsequent recombination in the quasineutral regions. A number of basic issues concerning the physics of the operation of solar cells still remain obscure. In this paper we briefly discussed some of those unsolved basic problems: need for not unphysical models of the carrier recombination and revision of the QN concept. In conventional solar cells recombination of photogenerated charge carriers plays a major limiting role in the cell efficiency. High quality thin-film solar cells may overcome this limit if the minority diffusion lengths become large as compared to the cell dimensions, but, strikingly, the conventional model fails to describe the cell electric behavior under these conditions. A new formulation of the basic equations describing charge carrier transport in the cell along with a set of boundary conditions is presented. An analytical closed-form solution is obtained under a linear approximation. In the new framework given, the calculation of the open-circuit voltage of the solar cell diode does not lead to unphysical results. 


\section{Acknowledgments}

J. E. Velazquez-Perez wants to thank Ministerio de Ciencia e Innovación (MICINN) and Junta de Castilla y León for partial financial support under Grants TEC2008-02281 and SA061A09. Yu. G. Gurevich wants to thank CONACYTMéxico for partial financial support.

\section{References}

[1] P. T. Landsberg, Recombination in Semiconductors, Cambridge University, Cambridge, UK, 1991.

[2] I. N. Volovichev, G. N. Logvinov, O. Y. Titov, and Y. G. Gurevich, "Recombination and lifetimes of charge carriers in semiconductors," Journal of Applied Physics, vol. 95, no. 8, pp. 4494-4496, 2004.

[3] Yu. G. Gurevich, J. E. Velazquez-Perez, G. Espejo-Lopez, I. N. Volovichev, and O. Y. Titov, "Transport of nonequilibrium carriers in bipolar semiconductors," Journal of Applied Physics, vol. 101, no. 2, Article ID 023705, 8 pages, 2007.

[4] Y. G. Gurevich and I. N. Volovichev, "Forgotten mechanism of nonlinearity in the theory of hot electrons," Physical Review B, vol. 60, no. 11, pp. 7715-7717, 1999.

[5] I. N. Volovichev, J. E. Velazquez-Perez, and Yu. G. Gurevich, "Transport boundary condition for semiconductor structures," Solid-State Electronics, vol. 52, no. 11, pp. 1703-1709, 2008.

[6] K. Seeger, Semiconductor Physics, Springer, Berlin, Germany, 1985.

[7] O. Yu. Titov, J. Giraldo, and Yu. G. Gurevich, "Boundary conditions in an electric current contact," Applied Physics Letters, vol. 80, no. 17, p. 3108, 2002.

[8] I. N. Volovichev, J. E. Velazquez-Perez, and Y. G. Gurevich, "Transport boundary conditions for solar cells," Solar Energy Materials and Solar Cells, vol. 93, no. 1, pp. 6-10, 2009.

[9] Y. G. Gurevich, G. N. Logvinov, J. E. Velazquez, and O. Y. Titov, "Transport and recombination in solar cells: new perspectives," Solar Energy Materials and Solar Cells, vol. 91, no. 15-16, pp. 1408-1411, 2007.

[10] M. A. Green, "Thin-film solar cells: review of materials, technologies and commercial status," Journal of Materials Science, vol. 18, supplement 1, pp. S15-S19, 2007.

[11] J. Singh, Semiconductor Devices: Basic Principles, John Wiley \& Sons, New York, NY, USA, 2000.

[12] A. Luque and S. Hegedus, Eds., Handbook of Photovoltaic Science and Engineering, John Wiley \& Sons, Chichester, UK, 2003.

[13] Sentaurus Device User Guide, Synopsys, Mountain View, Calif, USA, 2009.

[14] R. B. Bergmann, "Crystalline Si thin-film solar cells: a review," Applied Physics A, vol. 69, no. 2, pp. 187-194, 1999.

[15] J. E. Velázquez-Pérez and Y. G. Gurevich, "Transport and recombination in solar cells, new boundary conditions and perspectives," in Proceedings of the 25th European Photovoltaic Solar Energy Conference and Exhibition/5th World Conference on Photovoltaic Energy Conversion, Valencia, Spain, September 2010.

[16] Yu. G. Gurevich, G. N. Logvinov, I. N. Volovichev, G. Espejo, O. Yu. Titov, and A. Meriuts, "The role of nonequilibrium carriers in the formation of thermo-E.M.F. in bipolar semiconductors," Physica Status Solidi B, vol. 231, no. 1, pp. 278-293, 2002. 


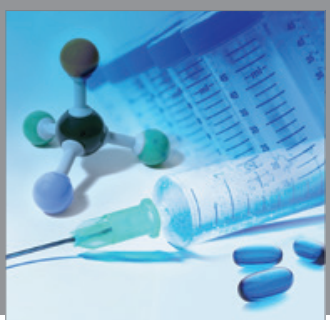

International Journal of

Medicinal Chemistry

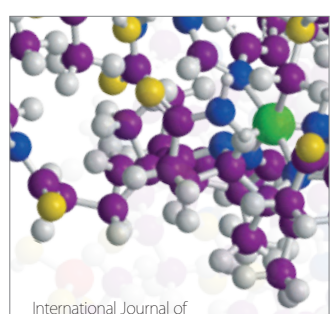

Carbohydrate Chemistry

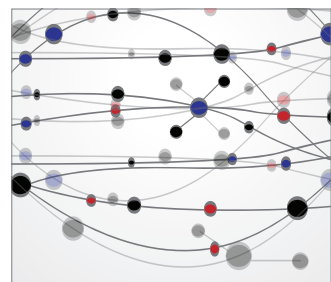

The Scientific World Journal
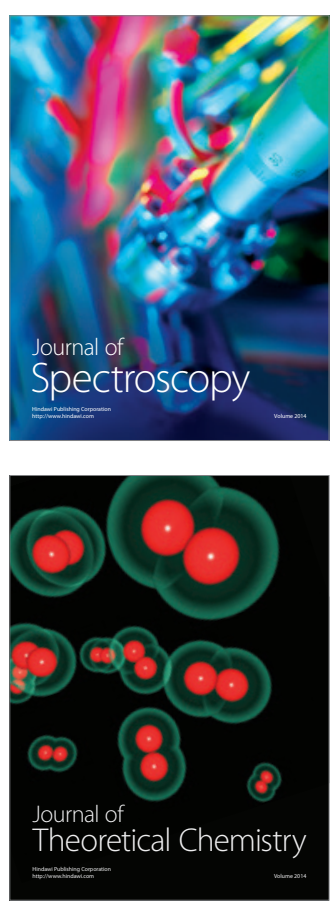
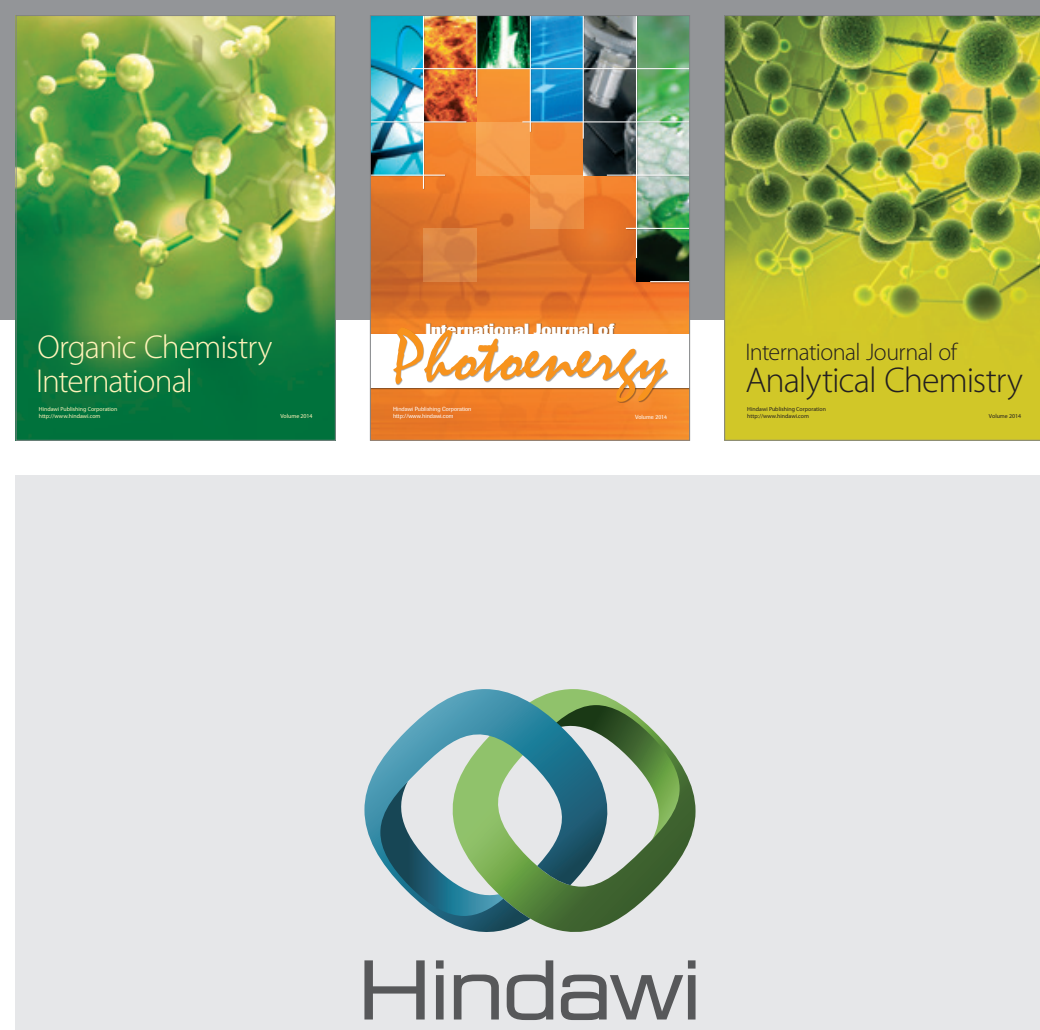

Submit your manuscripts at

http://www.hindawi.com
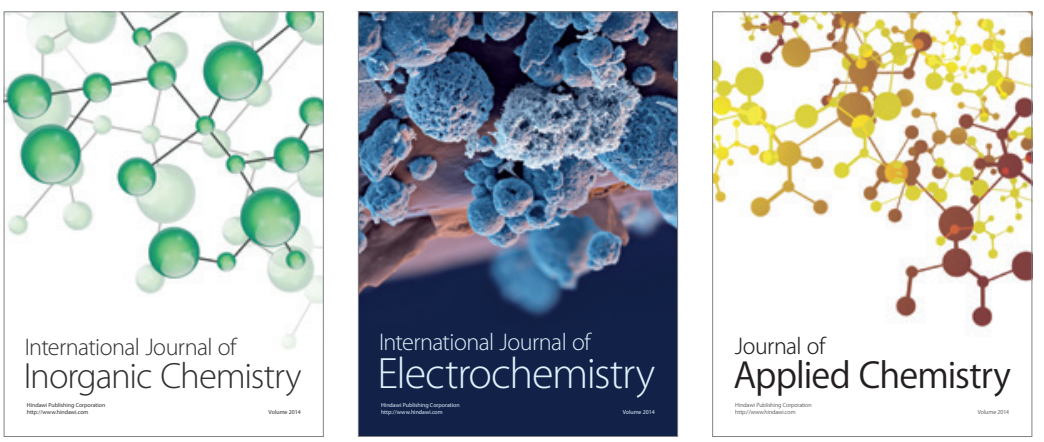

Journal of

Applied Chemistry
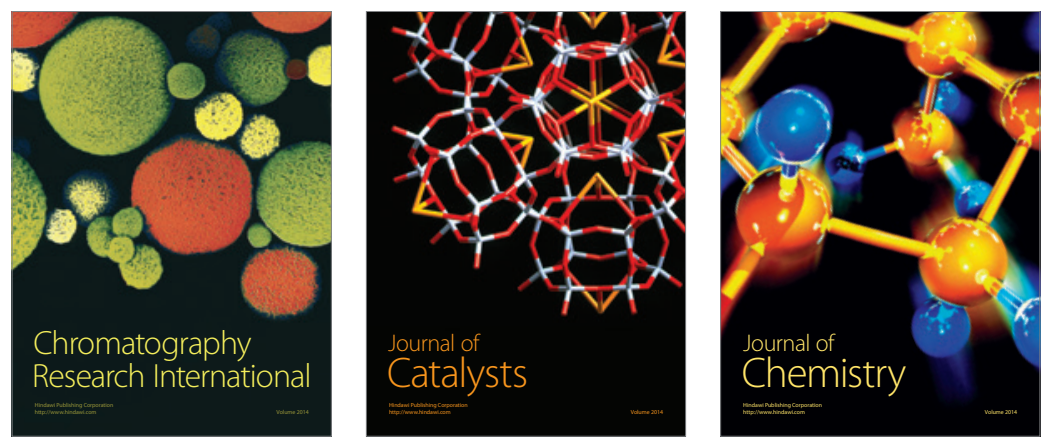
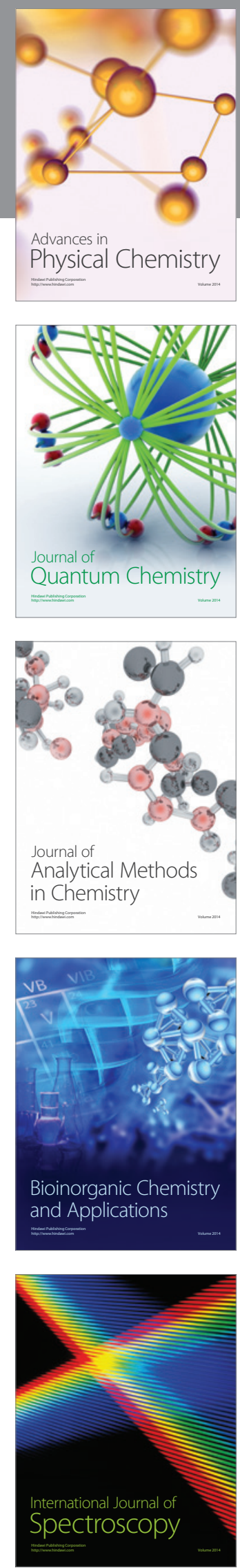